\title{
Performance of Generalized Multicarrier DS-CDMA Using Various Chip Waveforms
}

\author{
Lie-Liang Yang, Senior Member, IEEE, and Lajos Hanzo, Senior Member, IEEE
}

\begin{abstract}
In this letter, we extend our investigation of generalized multicarrier direct-sequence code-division multiple access by considering two additional types of chip waveforms, namely, the time-domain half-sine and raised-cosine chip waveforms, in addition to the rectangular chip waveform. A range of closed-form equations are provided for quantifying the effect of both the multipath interference and the multiuser interference, when using partially overlapping subcarriers. These closed-form formulas allow us to evaluate the bit-error rate performance of arbitrary code-division multiple access schemes using overlapping subbands with the aid of the standard Gaussian approximation.
\end{abstract}

Index Terms-Code-division multiple access (CDMA), multicarrier, frequency-selective fading.

\section{INTRODUCTION}

$\mathbf{I}$ $\mathrm{N}$ [1], A GENERALIZED multicarrier direct-sequence code-division multiple access (MC DS-CDMA) scheme using rectangular chip waveforms has been investigated, when communicating over frequency-selective Nakagami- $m$ fading channels [2], [3]. As argued in [1], in generalized MC DS-CDMA, the spacing between two adjacent subcarriers is a variable, allowing us to gain insight into the effects of the subcarrier spacing on the bit-error rate (BER) performance. This generalized MC DS-CDMA scheme includes the subclasses of multitone DS-CDMA [4] and orthogonal MC DS-CDMA [5] as special cases.

In this letter, we extend our investigations presented in [1] by considering two additional chip waveforms, namely, the timedomain half-sine and raised-cosine chip waveforms, in addition to the rectangular chip waveform. We provide the second-order statistics for the multipath interference (MPI) or multiuser interference (MUI), when the subcarrier signals are partially overlapped. A range of closed-form equations are obtained in the context of the chip waveforms considered. These closed-form equations allow us to evaluate the BER performance of CDMA systems using overlapping subbands [1], [4]-[7], with the aid of the standard Gaussian approximation.

Paper approved by D. I. Kim, the Editor for Spread Spectrum Transmission and Access of the IEEE Communications Society. Manuscript received November 30, 2001; revised June 11, 2002 and August 22, 2002. This work has been funded in the framework of the IST project IST-1999-12070 TRUST, which is partly funded by the European Union.

The authors are with the Department of Electronics and Computer Science, University of Southampton, Highfield Southampton SO17 1BJ, U.K. (e-mail 1ly@ecs.soton.ac.uk; 1h@ecs.soton.ac.uk).

Digital Object Identifier 10.1109/TCOMM.2003.811385

\section{RECEIVED SignALS AND DECISION VARIABLES}

In [1], we considered an asynchronous generalized MC DS-CDMA scheme, which supports $K$ users transmitting over a dispersive frequency-selective Nakagami- $m$ fading channel. The received signal is expressed as

$$
\begin{array}{r}
r(t)=\sum_{k=1}^{K} \sum_{u=1}^{U} \sum_{l_{p}=0}^{L_{p}-1} \sqrt{2 P} \alpha_{u l_{p}}^{(k)} b_{k u}\left(t-\tau_{k l_{p}}\right) c_{k}\left(t-\tau_{k l_{p}}\right) \\
\times \cos \left(2 \pi f_{u} t+\theta_{u l_{p}}^{(k)}\right)+n(t)
\end{array}
$$

where the following notations are used:
$K$

$U$

$f_{u}$

$T_{s}$

$T_{c}$

$N_{e}=T_{s} / T_{c}$

$n(t)$

P

$L_{p}$

$\alpha_{u l_{p}}^{(k)}$

$\tau_{k l_{p}}$ number of users;

number of subcarriers;

$u$ th subcarrier frequency;

symbol duration of the MC

DS-CDMA signal;

chip duration of the DS spreading sequences associated with each subcarrier;

spreading gain of each subcarrier signal;

additive white Gaussian noise (AWGN) with two-sided power spectrum density of $N_{0} / 2$;

average received power of each subcarrier signal;

number of resolvable paths of each subcarrier conveying a DS-CDMA signal;

Nakagami- $m$ distributed channel fading amplitude [[1], (8)]; multipath signal delay associated with asynchronous transmission and propagation, which is an independently and identically distributed (i.i.d.) uniform variable in $\left[0, T_{s}\right]$;

phase angle introduced in the carrier modulation and propagation processes, which is an i.i.d. uniform variable in $[0,2 \pi]$;

$b_{k u}(t) \quad$ binary data stream's waveform; $c_{k}(t)=\quad$ binary spreading sequence's $\sum_{j=-\infty}^{\infty} c_{k j} \psi\left(t-j T_{c}\right) \quad$ waveform, where $\psi(t)$ is the chip waveform.

Let the first user associated with $k=1$ be the user of interest, and consider the correlator-based RAKE receiver in conjunction with maximum ratio combining (MRC) [1]. We assume that the 
first $L, 1 \leq L \leq L_{p}$ number of resolvable paths are combined by the receiver. Consequently, as shown in [1], the decision variable $Z_{v}$ of the zeroth data bit corresponding to the $v$ th subcarrier of the reference user can be expressed as

$$
Z_{v}=\sum_{l=0}^{L-1} Z_{v l}, \quad v=1,2, \ldots, U
$$

where $Z_{v l}$ can be written as [1]

$$
\left.\begin{array}{rl}
Z_{v l}=\sqrt{\frac{P}{2}} T_{s}\left\{D_{v l}+N_{v l}+\sum_{\substack{l_{p}=0 \\
l_{p} \neq l}}^{L_{p}-1} I_{l}^{(s)}+\sum_{\substack{u=1 \\
u \neq v}}^{U} \sum_{l_{p}=0}^{L_{p}-1} I_{p}^{(s)}\right. \\
& +\sum_{k=2}^{K} \sum_{l_{p}=0}^{L_{p}-1} I_{l}^{(k)}+\sum_{k=2}^{K} \sum_{\substack{u=1 \\
u \neq v}}^{U} \sum_{l_{p}=0}^{L_{p}-1} I_{2}^{(k)}
\end{array}\right\}
$$

where $N_{v l}$ is a Gaussian random variable having zero mean and a variance of $\alpha_{v l}^{2} N_{0} / 2 E_{b}$, with $E_{b}=P T_{s}$ denoting the energy per bit, while $D_{v l}=b_{v}[0] \alpha_{v l}^{2}$ is the desired output. Furthermore, in (3), $Z_{v l}$ contains four types of interference.

- $I_{1}^{(s)}$ is the MPI contributed by the path $l_{p}, l_{p}=$ $0,1, \ldots, L_{p}-1$ and $l_{p} \neq l$ of the reference user associated with $k=1$, and with the same subcarrier of index $u=v$ as the reference user.

- $I_{2}^{(s)}$ is the MPI contributed by the path $l_{p}, l_{p}=$ $0, \ldots, L_{p}-1, l_{p} \neq l$, associated with the subcarriers $u$, $u=1,2, \ldots, U, u \neq v$ of the reference user.

- $I_{1}^{(k)}$ is the MUI due to the path $l_{p}, l_{p}=0,1, \ldots, L_{p}-1$ associated with the subcarrier $u=v$ engendered by the interfering users, $k=2,3, \ldots, K$.

- $I_{2}^{(k)}$ is the MUI due to the path $l_{p}, l_{p}=0, \ldots, L_{p}-1$ induced by the subcarrier $u, u=1, \ldots, U$ and $u \neq v$ of the interfering user $k, k=2,3, \ldots, K$.

Since random spreading sequences and an arbitrary spacing of $\Delta=\lambda / T_{s}$ between two adjacent subcarriers were assumed, it has been demonstrated in [1] that $I_{1}^{(s)}, I_{2}^{(s)}$, and $I_{1}^{(k)}$ constitute special cases of $I_{2}^{(k)}$, where $I_{2}^{(k)}$ can be expressed as

$$
\begin{aligned}
I_{2}^{(k)}=\frac{\alpha_{u l_{p}}^{(k)} \alpha_{v l}}{T_{s}}\left[b_{k_{u}}[-1]\right. & R_{k}\left(\tau_{k l_{p}}, \varphi_{u l_{p}}^{(k)}, u, v\right) \\
& \left.+b_{k u}[0] \hat{R}_{k}\left(\tau_{k l_{p}}, \varphi_{u l_{p}}^{(k)}, u, v\right)\right]
\end{aligned}
$$

where $\varphi_{u l_{p}}^{(k)}=\theta_{u l_{p}}^{(k)}-\theta_{v l}$ is a random variable uniformly distributed in $[0,2 \pi]$. The associated partial cross-correlation functions in (4) are defined by [1, eqs. (26), (27)].

\section{INTERFERENCE ANALYSIS}

Based on the standard Gaussian approximation, it has been shown in [1] that, for a given $\alpha_{v l}$ value, the MUI term $I_{2}^{(k)}$ of (4) can be approximated as a Gaussian random variable having zero mean and a variance given by

$$
\operatorname{Var}\left[I_{2}^{(k)}\right]=\Omega_{u l_{p}}^{(k)} \alpha_{v i}^{2} \mathcal{I}_{2}^{(k)}
$$

where $\Omega_{u l_{p}}^{(k)}=E\left[\left(\alpha_{u l_{p}}^{(k)}\right)^{2}\right]$, and

$$
\mathcal{I}_{2}^{(k)}=\frac{1}{T_{s}^{2}}\left\{E_{\tau, \varphi}\left[R_{k}^{2}(\tau, \varphi, u, v)\right]+E_{\tau, \varphi}\left[\hat{R}_{k}^{2}(\tau, \varphi, u, v)\right]\right\}
$$

where the superscript and subscript associated with the delay $\tau_{k l_{p}}$ and the phase angle $\varphi_{u l_{p}}^{(k)}$ have been ignored for simplicity. In (6), $E_{\tau, \varphi}\left[R_{k}^{2}(\tau, \varphi, u, v)\right]$ and $E_{\tau, \varphi}\left[\hat{R}_{k}^{2}(\tau, \varphi, u, v)\right]$ constitute the second central moments of $R_{k}(\tau, \varphi, u, v)$ and $\hat{R}_{k}(\tau, \varphi, u, v)$ with respect to $\tau$ and $\varphi$. Furthermore, it can be readily demonstrated that for random spreading sequences, we have $E_{\tau, \varphi}\left[R_{k}^{2}(\tau, \varphi, u, v)\right]=E_{\tau, \varphi}\left[\hat{R}_{k}^{2}(\tau, \varphi, u, v)\right]$ and

$$
\begin{aligned}
E_{\tau, \varphi}\left[R_{k}^{2}(\tau, \varphi, u, v)\right]= & \frac{\left(N_{e}+1\right)}{2} E_{\tau_{c}, \varphi}\left[R_{\psi}^{2}\left(\tau_{c}, \varphi, u, v\right)\right] \\
& +\frac{\left(N_{e}-1\right)}{2} E_{\tau_{c}, \varphi}\left[\hat{R}_{\psi}^{2}\left(\tau_{c}, \varphi, u, v\right)\right]
\end{aligned}
$$

where $E_{\tau_{c}, \varphi}\left[R_{\psi}^{2}\left(\tau_{c}, \varphi, u, v\right)\right]$ and $E_{\tau_{c}, \varphi}\left[\hat{R}_{\psi}^{2}\left(\tau_{c}, \varphi, u, v\right)\right]$ represent the second central moments of the extended partial autocorrelation functions $R_{\psi}\left(\tau_{c}, \varphi, u, v\right)$ and $\hat{R}_{\psi}\left(\tau_{c}, \varphi, u, v\right)$ of the chip waveforms, with respect to $\tau_{c}$ and $\varphi$, respectively. The $e x$ tended partial autocorrelation functions of the chip waveforms are defined as

$$
\begin{aligned}
R_{\psi}\left(\tau_{c}, \varphi, u, v\right)= & \int_{0}^{\tau_{c}} \psi(t) \psi\left(t+T_{c}-\tau_{c}\right) \\
& \times \cos \left(\frac{2 \pi \lambda(u-v) t}{T_{s}}+\varphi\right) d t \\
\hat{R}_{\psi}\left(\tau_{c}, \varphi, u, v\right)= & \int_{\tau_{c}}^{T_{c}} \psi(t) \psi\left(t-T_{c}-\tau_{c}\right) \\
& \times \cos \left(\frac{2 \pi \lambda(u-v) t}{T_{s}}+\varphi\right) d t
\end{aligned}
$$

Below, we provide the expressions of $E_{\tau_{c}, \varphi}\left[R_{\psi}^{2}\left(\tau_{c}, \varphi, u, v\right)\right]$, and $E_{\tau_{c}, \varphi}\left[\hat{R}_{\psi}^{2}\left(\tau_{c}, \varphi, u, v\right)\right]$ for the three classes of chip waveforms considered without detailed derivations.

\section{A. Rectangular Chip Waveform}

For a rectangular chip waveform of $\psi(t)=P_{T_{c}}(t)$, we have $P_{T_{c}}(t)=1$ for $0 \leq t \leq T_{c}$ and $P_{T_{c}}(t)=0$, otherwise. If $\lambda(u-v) \neq 0$, then we have

$$
\begin{aligned}
& E_{\tau_{c}, \varphi}\left[R_{\psi}^{2}\left(\tau_{c}, \varphi, u, v\right)\right] \\
& \quad=E_{\tau_{c}, \varphi}\left[\hat{R}_{\psi}^{2}\left(\tau_{c}, \varphi, u, v\right)\right] \\
& \quad=\frac{T_{s}^{2}}{4 \pi^{2} \lambda^{2}(u-v)^{2}}\left[1-\operatorname{sinc}\left(\frac{2 \pi \lambda(u-v)}{N_{e}}\right)\right] .
\end{aligned}
$$

If $\lambda(u-v)=0$, then we arrive at

$$
E_{\tau_{c}, \varphi}\left[R_{\psi}^{2}\left(\tau_{c}, \varphi\right)\right]=E_{\tau_{c}, \varphi}\left[\hat{R}_{\psi}^{2}\left(\tau_{c}, \varphi\right)\right]=\frac{T_{c}^{2}}{6}
$$




\section{B. Half-Sine Chip Waveform}

For a half-sine chip waveform, we have $\psi(t)=$ $\sqrt{2} \sin \left(\pi t / T_{c}\right) P_{T_{c}}(t)$. Then, if $|\lambda(u-v)| \neq 0, N_{e}$, then we get

$$
\begin{aligned}
& E_{\tau_{c}, \varphi}\left[R_{\psi}^{2}\left(\tau_{c}, \varphi, u, v\right)\right] \\
& =E_{\tau_{c}, \varphi}\left[\hat{R}_{\psi}^{2}\left(\tau_{c}, \varphi, u, v\right)\right] \\
& =\frac{T_{s}^{2}}{8 \pi^{2}}\left[\frac{1}{\lambda^{2}(u-v)^{2}}+\frac{1}{2\left[N_{e}+\lambda(u-v)\right]^{2}}\right. \\
& +\frac{1}{2\left[N_{e}-\lambda(u-v)\right]^{2}}-\frac{1}{\lambda(u-v)\left[N_{e}+\lambda(u-v)\right]} \\
& +\frac{1}{\lambda(u-v)\left[N_{e}-\lambda(u-v)\right]} \\
& -\left(\frac{1}{\lambda^{2}(u-v)^{2}}-\frac{1}{\lambda(u-v)\left[N_{e}+\lambda(u-v)\right]}\right. \\
& +\frac{1}{\lambda(u-v)\left[N_{e}-\lambda(u-v)\right]} \\
& \left.-\frac{1}{N_{e}^{2}-\lambda^{2}(u-v)^{2}}\right) \operatorname{sinc}\left(\frac{2 \pi \lambda(u-v)}{N_{e}}\right) \\
& -\left(\frac{1}{2 \lambda^{2}(u-v)^{2}}+\frac{1}{2\left[N_{e}+\lambda(u-v)\right]^{2}}\right. \\
& \left.-\frac{1}{\lambda(u-v)\left[N_{e}+\lambda(u-v)\right]}\right) \\
& \times \operatorname{sinc}\left(\frac{2 \pi\left[N_{e}+\lambda(u-v)\right]}{N_{e}}\right) \\
& -\left(\frac{1}{2 \lambda^{2}(u-v)^{2}}+\frac{1}{2\left[N_{e}-\lambda(u-v)\right]^{2}}\right. \\
& \left.+\frac{1}{\lambda(u-v)\left[N_{e}-\lambda(u-v)\right]}\right) \\
& \left.\times \operatorname{sinc}\left(\frac{2 \pi\left[N_{e}-\lambda(u-v)\right]}{N_{e}}\right)\right] .
\end{aligned}
$$

If $\lambda(u-v)=0$, then we have

$$
E_{\tau_{c}, \varphi}\left[R_{\psi}^{2}\left(\tau_{c}, \varphi\right)\right]=E_{\tau_{c}, \varphi}\left[\hat{R}_{\psi}^{2}\left(\tau_{c}, \varphi\right)\right]=T_{c}^{2}\left(\frac{1}{12}+\frac{5}{8 \pi^{2}}\right) .
$$

Finally, if $|\lambda(u-v)|=N_{e}$, then we arrive at

$$
E_{\tau_{c}, \varphi}\left[R_{\psi}^{2}\left(\tau_{c}, \varphi\right)\right]=E_{\tau_{c}, \varphi}\left[\hat{R}_{\psi}^{2}\left(\tau_{c}, \varphi\right)\right]=T_{c}^{2}\left(\frac{1}{24}+\frac{5}{64 \pi^{2}}\right) .
$$

\section{Raised-Cosine Chip Waveform}

Finally, with respect to the raised-cosine chip waveform, we have $\psi(t)=\sqrt{2 / 3}\left[1-\cos \left(2 \pi t / T_{c}\right)\right] P_{T_{c}}(t)$ [8]. Consequently, if $|\lambda(u-v)| \neq 0, N_{e}, 2 N_{e}$, we have

$$
\begin{aligned}
& E_{\tau_{c}, \varphi}\left[R_{\psi}^{2}\left(\tau_{c}, \varphi, u, v\right)\right] \\
& \quad=E_{\tau_{c}, \varphi}\left[\hat{R}_{\psi}^{2}\left(\tau_{c}, \varphi, u, v\right)\right] \\
& \quad=\frac{T_{s}^{2}}{72 \pi^{2}}\left[\left(\frac{9}{\lambda^{2}(u-v)^{2}}+\frac{4}{\left[N_{e}+\lambda(u-v)\right]^{2}}+\frac{4}{\left[N_{e}-\lambda(u-v)\right]^{2}}\right.\right.
\end{aligned}
$$

$$
\begin{aligned}
& +\frac{1}{2\left[2 N_{e}+\lambda(u-v)\right]^{2}}+\frac{1}{2\left[2 N_{e}-\lambda(u-v)\right]^{2}} \\
& 10 \\
& -\overline{\lambda(u-v)\left[N_{e}+\lambda(u-v)\right]} \\
& +\frac{10}{\lambda(u-v)\left[N_{e}-\lambda(u-v)\right]} \\
& +\frac{1}{\lambda(u-v)\left[2 N_{e}+\lambda(u-v)\right]} \\
& -\frac{1}{\lambda(u-v)\left[2 N_{e}-\lambda(u-v)\right]}-\frac{4}{N_{e}^{2}-\lambda^{2}(u-v)^{2}} \\
& -\frac{2}{\left[N_{e}+\lambda(u-v)\right]\left[2 N_{e}+\lambda(u-v)\right]} \\
& \left.-\frac{2}{\left[N_{e}-\lambda(u-v)\right]\left[2 N_{e}-\lambda(u-v)\right]}\right) \\
& -\left(\frac{9}{\lambda^{2}(u-v)^{2}}+\frac{2}{\left[N_{e}+\lambda(u-v)\right]^{2}}\right. \\
& +\frac{2}{\left[N_{e}-\lambda(u-v)\right]^{2}}-\frac{10}{\lambda(u-v)\left[N_{e}+\lambda(u-v)\right]} \\
& +\frac{10}{\lambda(u-v)\left[N_{e}-\lambda(u-v)\right]} \\
& +\frac{1}{\lambda(u-v)\left[2 N_{e}+\lambda(u-v)\right]} \\
& -\frac{1}{\lambda(u-v)\left[2 N_{e}-\lambda(u-v)\right]}-\frac{8}{N_{e}^{2}-\lambda^{2}(u-v)^{2}} \\
& +\frac{2}{\left[N_{e}+\lambda(u-v)\right]\left[2 N_{e}-\lambda(u-v)\right]} \\
& +\frac{2}{\left[N_{e}-\lambda(u-v)\right]\left[2 N_{e}+\lambda(u-v)\right]} \\
& \left.-\frac{1}{\left[4 N_{e}^{2}-\lambda^{2}(u-v)^{2}\right]}\right) \times \operatorname{sinc}\left(\frac{2 \pi \lambda(u-v)}{N_{e}}\right) \\
& -\left(\frac{4}{\lambda^{2}(u-v)^{2}}+\frac{4}{\left[N_{e}+\lambda(u-v)\right]^{2}}\right. \\
& -\frac{10}{\lambda(u-v)\left[N_{e}+\lambda(u-v)\right]} \\
& +\frac{2}{\lambda(u-v)\left[N_{e}-\lambda(u-v)\right]} \\
& +\frac{4}{\lambda(u-v)\left[2 N_{e}+\lambda(u-v)\right]} \\
& -\frac{4}{\left[N_{e}^{2}-\lambda^{2}(u-v)^{2}\right]} \\
& -\frac{2}{\left[N_{e}+\lambda(u-v)\right]\left[2 N_{e}+\lambda(u-v)\right]} \\
& \left.+\frac{2}{\left[N_{e}-\lambda(u-v)\right]\left[2 N_{e}+\lambda(u-v)\right]}\right) \\
& \times \operatorname{sinc}\left(\frac{2 \pi\left[N_{e}+\lambda(u-v)\right]}{N_{e}}\right) \\
& -\left(\frac{4}{\lambda^{2}(u-v)^{2}}+\frac{4}{\left[N_{e}-\lambda(u-v)\right]^{2}}\right. \\
& +\frac{10}{\lambda(u-v)\left[N_{e}-\lambda(u-v)\right]} \\
& -\frac{2}{\lambda(u-v)\left[N_{e}+\lambda(u-v)\right]}
\end{aligned}
$$




$$
\begin{aligned}
& -\frac{4}{\lambda(u-v)\left[2 N_{e}-\lambda(u-v)\right]} \\
& -\frac{4}{\left[N_{e}^{2}-\lambda^{2}(u-v)^{2}\right]} \\
& +\frac{2}{\left[N_{e}+\lambda(u-v)\right]\left[2 N_{e}-\lambda(u-v)\right]} \\
& \left.-\frac{2}{\left[N_{e}-\lambda(u-v)\right]\left[2 N_{e}-\lambda(u-v)\right]}\right) \\
& \times \operatorname{sinc}\left(\frac{2 \pi\left[N_{e}-\lambda(u-v)\right]}{N_{e}}\right) \\
& -\left(\frac{1}{2 \lambda^{2}(u-v)^{2}}+\frac{2}{\left[N_{e}+\lambda(u-v)\right]^{2}}\right. \\
& +\frac{1}{2\left[2 N_{e}+\lambda(u-v)\right]^{2}} \\
& 2 \\
& -\overline{\lambda(u-v)\left[N_{e}+\lambda(u-v)\right]} \\
& +\frac{1}{\lambda(u-v)\left[2 N_{e}+\lambda(u-v)\right]} \\
& \left.-\frac{2}{\left[N_{e}+\lambda(u-v)\right]\left[2 N_{e}+\lambda(u-v)\right]}\right) \\
& \times \operatorname{sinc}\left(\frac{2 \pi\left[2 N_{e}+\lambda(u-v)\right]}{N_{e}}\right) \\
& -\left(\frac{1}{2 \lambda^{2}(u-v)^{2}}+\frac{2}{\left[N_{e}-\lambda(u-v)\right]^{2}}\right. \\
& +\frac{1}{2\left[2 N_{e}-\lambda(u-v)\right]^{2}} \\
& +\frac{2}{\lambda(u-v)\left[N_{e}-\lambda(u-v)\right]} \\
& -\frac{1}{\lambda(u-v)\left[2 N_{e}-\lambda(u-v)\right]} \\
& \left.-\frac{2}{\left[N_{e}-\lambda(u-v)\right]\left[2 N_{e}-\lambda(u-v)\right]}\right) \\
& \left.\times \operatorname{sinc}\left(\frac{2 \pi\left[2 N_{e}-\lambda(u-v)\right]}{N_{e}}\right)\right] \text {. }
\end{aligned}
$$

If $\lambda(u-v)=0$, then we have

$E_{\tau_{c}, \varphi}\left[R_{\psi}^{2}\left(\tau_{c}, \varphi\right)\right]=E_{\tau_{c}, \varphi}\left[\hat{R}_{\psi}^{2}\left(\tau_{c}, \varphi\right)\right]=T_{c}^{2}\left(\frac{1}{12}+\frac{315}{864 \pi^{2}}\right)$.

If $|\lambda(u-v)|=N_{e}$, then we get

$E_{\tau_{c}, \varphi}\left[R_{\psi}^{2}\left(\tau_{c}, \varphi\right)\right]=E_{\tau_{c}, \varphi}\left[\hat{R}_{\psi}^{2}\left(\tau_{c}, \varphi\right)\right]=T_{c}^{2}\left(\frac{1}{27}+\frac{35}{162 \pi^{2}}\right)$.

Finally, if $|\lambda(u-v)|=2 N_{e}$, then we arrive at

$$
\begin{aligned}
E_{\tau_{c}, \varphi}\left[R_{\psi}^{2}\left(\tau_{c}, \varphi\right)\right] & =E_{\tau_{c}, \varphi}\left[\hat{R}_{\psi}^{2}\left(\tau_{c}, \varphi\right)\right] \\
& =T_{c}^{2}\left(\frac{1}{216}+\frac{245}{20736 \pi^{2}}\right) .
\end{aligned}
$$

Based on the above second central moment values of the partial autocorrelation functions with respect to the rectangular, half-sine, and raised-cosine chip waveforms, the corresponding expectation values in $E_{\tau, \varphi}\left[R_{k}^{2}(\tau, \varphi, u, v)\right]$ of (7) as well as $E_{\tau, \varphi}\left[\hat{R}_{k}^{2}(\tau, \varphi, u, v)\right]=E_{\tau, \varphi}\left[R_{k}^{2}(\tau, \varphi, u, v)\right]$ can be obtained.

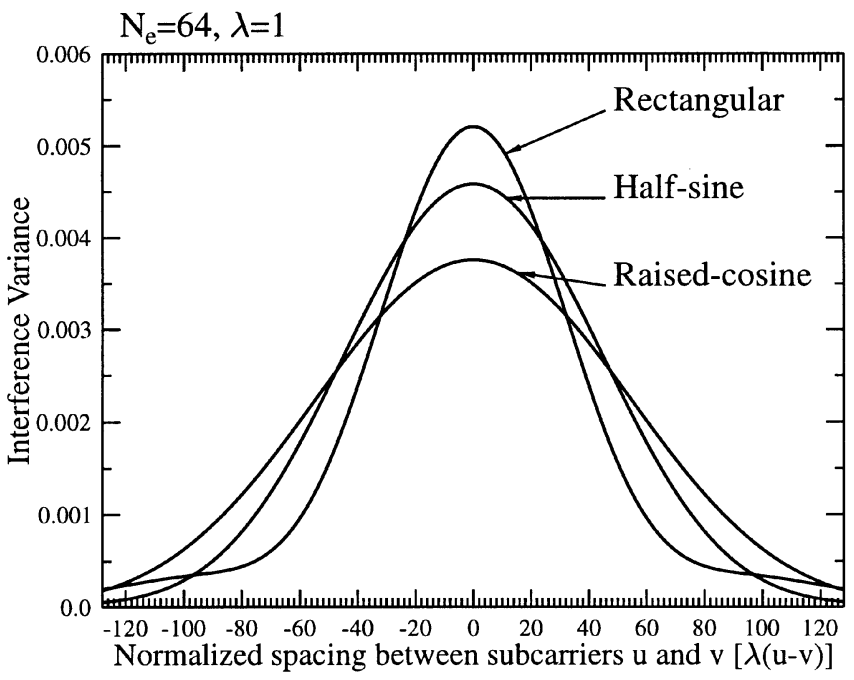

Fig. 1. Interference power $\mathcal{I}_{2}^{(k)}$ of (6) versus the normalized spacing, $\lambda(u-v)$ between two subcarrier frequencies $f_{u}$ and $f_{v}$, with respect to the rectangular, half-sine, and raised-cosine chip waveforms.

Upon substituting these expectation values into (6) and (5), the variance of $\mathcal{I}_{2}^{(k)}$ and $I_{2}^{(k)}$ corresponding to the rectangular, half-sine, or raised-cosine chip waveforms can be evaluated. Specifically, for the case of $\lambda(u-v)=0$, we have

$\mathcal{I}_{2}^{(k)}=\mathcal{I}_{1}^{(k)}=\mathcal{I}_{1}^{(s)}=\mathcal{I}_{0}= \begin{cases}\frac{1}{3 N_{e}}, & \text { Rectangular } \\ \frac{1}{6 N_{e}}+\frac{5}{4 N_{e} \pi^{2}}, & \text { Half-sine } \\ \frac{1}{6 N_{e}}+\frac{315}{432 N_{e} \pi^{2}}, & \text { Raised-cosine }\end{cases}$

which are some of the typical variance values for the MUI in the context of single-carrier DS-CDMA schemes.

Having obtained the statistics of both the MPI as well as the MAI, the average BER of the generalized MC DS-CDMA system communicating over multipath Nakagami- $m$ fading channels can be estimated using [1, eq. (47)], with respect to the three classes of chip waveforms considered.

\section{NUMERICAL RESULTS}

Fig. 1 gives us an insight into the interference behavior for different chip waveforms associated with different subcarrier spacings. Let us assume that $f_{v}$ is one of the subcarrier frequencies of the reference signal, while $f_{u}$ is one of the subcarrier frequencies used by the interfering signals. According to the results of Fig. 1, we observe that, for all the chip waveforms considered, the interference power decreases, when increasing the absolute spacing value of $|\lambda(u-v)|$. If we have $\lambda(u-v)=0$, which implies that $f_{u}=f_{v}$, the spreading sequences using a rectangular chip waveform impose the highest interference power, while using a raised-cosine chip waveform results in the lowest interference power. When increasing the spacing $|\lambda(u-v)|$, we can observe that there exists a spacing range, where the rectangular chip waveform outperforms both the half-sine and the raised-cosine chip waveforms. The results of Fig. 1 demonstrate that the interference power at the point of $\lambda(u-v)=0$ is more than 20 times higher than that at the point of $\lambda(u-v)=2 N_{e}=128$. 


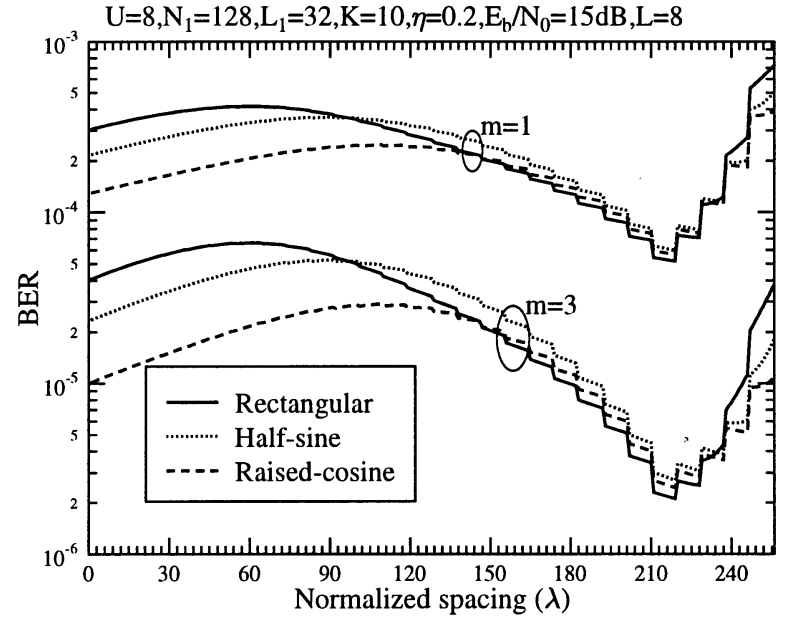

Fig. 2. BER versus the normalized subcarrier spacing, $\lambda$ for the generalized MC DS-CDMA system communicating over the multipath Nakagami- $m$ fading channel using the fading parameters of $m=1$ and $m=3$. The results were computed by assuming that the maximum multipath combining capability was $L=8$, i.e., that the receiver was capable of combining, at most, eight resolvable paths due to implementation complexity limitations.

Therefore, we can infer that in a MC DS-CDMA system, the MPI and the MUI are mainly contributed by the specific subcarrier signals having overlapping main lobes.

The influence of the normalized subcarrier spacing, $\lambda$, on the average BER of the generalized MC DS-CDMA system is shown in Fig. 2, where we assumed that the fading parameters of $m=1$ and 3, and that the receiver was capable of combining, at most, $L=8$ resolvable paths in Fig. 2. At the top of Fig. 2, $N_{1}$ and $L_{1}$ represent the spreading gain and the number of resolvable paths of a corresponding single-carrier DS-CDMA scheme using the same total bandwidth as the MC DS-CDMA arrangement. From the results of Fig. 2, we observe that for each of the chip waveforms considered, there exists an optimum value of $\lambda$, which will result in the minimum average BER. The optimum value of $\lambda$ is similar for all three types of chip waveforms. Specifically, the optimum value was around $\lambda=215$. Furthermore, from the results of Fig. 2, we observe that when the normalized spacing of $\lambda$ assumes a sufficiently high value, the MC DS-CDMA systems using any of the three chip waveforms studied achieved a similar BER performance.

\section{CONCLUSION}

In summary, the generalized MC DS-CDMA system of [1] has been investigated by assuming that the spreading sequences use various chip waveforms, namely, rectangular, half-sine, and raised-cosine time-domain chip waveforms. The effects of the chip waveforms and the spacing between two adjacent subcarriers on the performance of the generalized MC DS-CDMA systems have been evaluated. The results show that for a given subcarrier spacing between two adjacent subcarriers, there exists a corresponding best choice of the chip waveform.

\section{ACKNOWLEDGMENT}

The authors would like to acknowledge the contributions of their colleagues.

\section{REFERENCES}

[1] L.-L. Yang and L. Hanzo, "Performance of generalized multicarrier DS-CDMA over Nakagami- $m$ fading channels," IEEE Trans. Commun., vol. 50, pp. 914-925, June 2002.

[2] N. Nakagami, "The $m$-distribution, a general formula for intensity distribution of rapid fading," in Statistical Methods in Radio Wave Propagation, W. G. Hoffman, Ed. Oxford, U. K.: Pergamon, 1960.

[3] M.-S. Alouini and M. K. Simon, Digital Communication Over Fading Channels: A Unified Approach to Performance Analysis. New York: Wiley, 2000.

[4] L. Vandendorpe, "Multitone spread spectrum multiple access communications system in a multipath Rician fading channel," IEEE Trans. Veh Technol., vol. 44, pp. 327-337, Feb. 1995.

[5] E. A. Sourour and M. Nakagawa, "Performance of orthogonal multicarrier CDMA in a multipath fading channel," IEEE Trans. Commun., vol. 44, pp. 356-367, Mar. 1996.

[6] M. A. Laxpati and J. W. Gluck, "Optimization of hybrid SFH/DS MFSK link in the presence of worst case multitone jamming," IEEE Trans. Commun., vol. 43, pp. 2118-2156, June 1995.

[7] L.-L. Yang and L. Hanzo, "Overlapping $M$-ary frequency shift keying spread-spectrum multiple-access systems using random signature sequences," IEEE Trans. Veh. Technol., vol. 48, pp. 1984-1995, Nov. 1999.

[8] H. H. Nguyen and E. Shwedyk, "On error probability of DS-CDMA systems with arbitrary chip waveforms," IEEE Commun. Lett., vol. 5, pp. 78-80, Mar. 2001. 